International Journal of Engineering \& Technology, $7(2.5)(2018) 32-36$
International Journal of Engineering \& Technology
SPC
Website: www.sciencepubco.com/index.php/IJET
Research Paper

\title{
Analysis determinants of social media acceptance in higher educational institutes of Pakistan
}

\author{
Ali Raza ${ }^{1 *}$, ShahMurad Chandio', Najma Imtaiz Ali ${ }^{1}$, Zulfiqar Solangi ${ }^{1}$, Asadullah Shah ${ }^{1}$, Syed Faiz Ahmed ${ }^{2}$ \\ ${ }^{1}$ International Islamic University Malaysia, Malaysia \\ ${ }^{2}$ Universiti Kuala Lumpur, British Malaysian Institute \\ *Corresponding author E-mail: alirazarang@gmail.com
}

\begin{abstract}
Seen through the lens of the educational sector, social media grew to become a vital source of academic learning. The learning through social media occurs mainly through the collaborative approach to information sharing, where the web-based social networking sites provide the optimal platform for knowledge enhancing among colleagues, co-workers, and others. Developed economies have already recognized the significant value of learning through social media. However, developing economies such as Pakistan did not yet interpret future implications and real benefits of social media aided learning. This research focuses on determining significant factors through an integrated framework that features broadly recognized technology models such as Technology acceptance model (TAM) and Innovation diffusion theory (IDT). The subjects of the framework testing were students in higher education institutions that use social media, and the sample size was 350 students. Data analysis results, reached via SPSS software, were substantially in favour of extended model. Results reached through this study, in terms of factors with a significant influence on social media acceptance rate in Pakistani higher education institutions, are particularly crucial for students in the field of education, located in developing countries. This should assist the increasing acceptance and use of technological solutions, benefiting both faculty and students.
\end{abstract}

Keywords: Innovation Diffusion Theory, Technology Acceptance Model, Learning and Social Media.

\section{Introduction}

The widespread globalization, bolstered by electronic devices and easier communication, has turned the world into a global village, making things and people interconnected more than ever before. We are witnessing the revolutionary impact of social media on people's everyday life, changing the means of communicating with and keeping track of family and friends' movements and activities.

This study primarily focuses on the current and future social media usage in the Pakistani educational sector. It is estimated that $70 \%$ of Pakistani social media users consists of people aged under 25 [1]. The purpose of this study is to investigate the acceptance of social media among the population of university students. To identify significant factors related to the social media, a framework that integrates various prominent social theories and technology adoption, including TAM and IDT, is used. The goal is to identify the significant factors related to the acceptance of social media for educational purposes.

\section{Related work}

TAM was utilized to define the behaviour of computer usage. This framework or model is recognized as the imperative, most frequently cited model for understanding the human acceptance of information technology, also supported by extensive empirical evidence [2]. TAM framework suggests two external variables, professed ease of use and perceived utility, as two impact factors on two internal beliefs. PU is commonly reflecting a degree to which an individual believes that technological system/solution would enhance his/her work performance, while PEOU is defined as the degree of new technology ease of usage [3].

Disciplines such as marketing, communication, education, sociology, information technology and social media have largely benefited from diffusion of innovation [4, 5, 6]. In simple terms, innovation is defined as an idea, practice or object that an individual perceives as brand new, while process by which the members of a social system communicate an innovation over time through distinct channels is defined as diffusion [4].

\section{Proposed conceptual framework}

\subsection{Acceptance of social media}

In this research, dependent variable is defined as the acceptance of social media for education. In this context, social media is defined as the cluster of communication networks dedicated to sharing, interaction and collaboration among its users. There are numerous types of social media in the for of websites and applications such as social networks, social bookmarks, wikis, and micro blogs.

\subsection{Perceived usefulness}

PU is commonly reflecting a degree to which an individual believes that technological solution would enhance his/her work performance [7]. Thus, PU suggests that students' expectations were positive in regards to how the new technology adoption will reflect on their academic performance [8]. 


\subsection{Perceived use of ease}

PEOU is defined as the degree to which users will find new technology to remain effortless [7]. PEOU suggests that students have positive expectations towards how the new technology implementation will reflect on their academic performance. It is deemed by individuals that using technology will be user-friendly with minimized cognitive effort [8].

\subsection{Compatibility}

Compatibility describes the extent of individual's previous technological experience and positively correlates with acceptance of new information technology [9]. Thus, compatibility affects perceived usefulness and behavioural intention in direct and positive manner $[10,11]$.

\subsection{Complexity}

According to previous studies, complexity is found to be affecting negatively the intention of use and perceived usefulness [12]. Research revealed that the end user is less likely to use networking if end user is perceiving this system as [13].

\subsection{Relevance}

It is defining the degree to which the technology/system is matching tasks as accepted in the present environment and as identified through the task analysis. The effectiveness of information retrieval system can be measured as relevance includes both recall and precision factors [14]. Later, if relevant information is made available on a bigger scale through social media, the user will retrieve valuable information more easily.

\subsection{Self-efficacy}

Self-efficacy defines the extent to which people are willing to achieve a certain performance level and their judgement about it. This judgement of self-efficacy is important as it determines how much effort will the individual put if for a certain cause and how long will that effort endure [15].

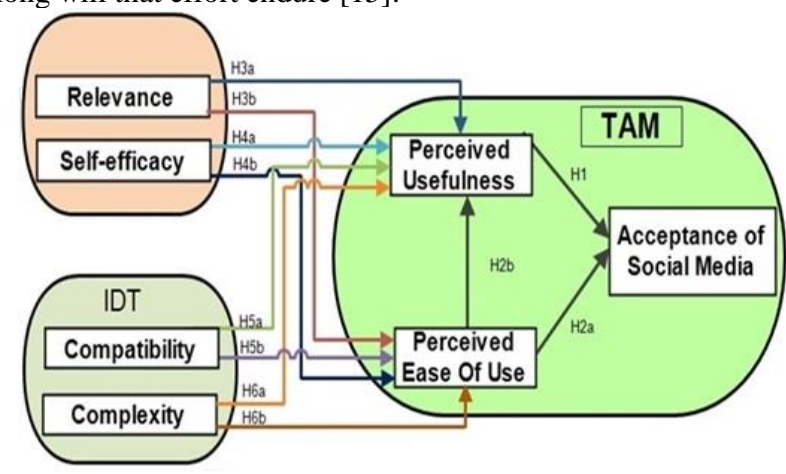

Fig. 1: Proposed research model for acceptance of social media. [16]

\section{Method}

In this sections, sample, measurement, analysis tools and techniques are discussed which are used to carry out the current research.

\section{Population and sampling frame}

In Pakistan, the total number of internet users are estimated to be approximately 30 million [1]. Furthermore, total number of student in universities estimated to be approximately 1.107 million the sampling frame of the current study are shown in Table 1.
Table 1: Universities in hyderabad, sindh, Pakistan

\begin{tabular}{lll}
\hline & Universities & No of students \\
\cline { 2 - 3 } 1 & $\begin{array}{l}\text { Mehran of engineering and technology } \\
\text { University of Sindh }\end{array}$ & 5200 \\
2 & $\begin{array}{l}\text { Liaquat university of medical health } \\
\text { science }\end{array}$ & 26500 \\
3 & Sindh agricultural university & 3800 \\
& $\quad$ Total & 6000 \\
& & 41500 \\
\hline
\end{tabular}

\section{Techniques and sample size}

The purpose of sampling, according to [17] is to collect information regarding population through utilization of the sample. The more a sample characterizes a population, the more generalized the assumptions become. Sampling is categorized into two major types: PS and NPS [18]. Data collection is done by using convenience sampling because; the researcher doesn't have access to every student list in every university due to privacy and security issue.

\section{Analysis tools}

To analyses the data (SPSS) was used for descriptive statistics, normality and EFA.

\subsection{Exploratory factor analysis}

Parasuraman [19] defined "EFA as multivariate statistic method that evaluates and examines data on a comparatively big variable set and then generates fewer factors, which are insert compounds of the initial variables, and thus the factors set takes info from given data set".

\section{Results}

Results obtained from current study are presented in this section.

\subsection{Demographic characteristics of participants}

Demographic characteristics of 350 respondents obtained from the study are presented here. Outcomes of participants' background information and personal information are shown in Table: 2 to Table 6.

Table 2: Usage of social media

\begin{tabular}{lllll}
\hline $\begin{array}{l}\text { How often do you use social } \\
\text { media? }\end{array}$ & $\begin{array}{l}\text { Fre- } \\
\text { quency }\end{array}$ & Percent & $\begin{array}{l}\text { Valid } \\
\text { Percent }\end{array}$ & $\begin{array}{l}\text { Cumula- } \\
\text { tive Per- } \\
\text { cent }\end{array}$ \\
\hline More than once a day & 137 & 39.1 & 39.1 & 39.1 \\
Once a day & 58 & 16.6 & 16.6 & 55.7 \\
Several times a week & 64 & 18.3 & 18.3 & 74.0 \\
Once a week & 44 & 12.6 & 12.6 & 86.6 \\
Several times a month & 27 & 7.7 & 7.7 & 94.3 \\
Once a month & 20 & 5.7 & 5.7 & 100.0 \\
Total & 350 & 100.0 & 100.0 & \\
\hline
\end{tabular}

Table 3: Purpose and qualification of participation

\begin{tabular}{lllll}
\hline $\begin{array}{l}\text { For what purpose do you } \\
\text { use social media? }\end{array}$ & \multicolumn{2}{l}{ FrequencyPercent } & $\begin{array}{l}\text { Valid } \\
\text { Percent }\end{array}$ & $\begin{array}{l}\text { Cumulative } \\
\text { Percent }\end{array}$ \\
\hline Education & 152 & 43.4 & 43.4 & 43.4 \\
Job & 12 & 3.4 & 3.4 & 46.9 \\
Social Activities & 171 & 48.9 & 48.9 & 95.7 \\
Other & 15 & 4.3 & 4.3 & 100.0 \\
Total & 350 & 100.0 & 100.0 & \\
\hline
\end{tabular}


Table 4: Gender based statistics

\begin{tabular}{lllll}
\hline & Frequency & Percent & $\begin{array}{l}\text { Valid Per- } \\
\text { cent }\end{array}$ & $\begin{array}{l}\text { Cumulative Per- } \\
\text { cent }\end{array}$ \\
\hline Male & 208 & 59.4 & 59.4 & 59.4 \\
Female & 141 & 40.3 & 40.3 & 99.7 \\
3 & 1 & .3 & .3 & 100.0 \\
Total & 350 & 100.0 & 100.0 & \\
\hline
\end{tabular}

Table 5: Education

\begin{tabular}{lllll}
\hline & $\begin{array}{l}\text { Fre- } \\
\text { quency }\end{array}$ & $\begin{array}{l}\text { Per- } \\
\text { cent }\end{array}$ & $\begin{array}{l}\text { Valid } \\
\text { Per- } \\
\text { cent }\end{array}$ & $\begin{array}{l}\text { Cumula- } \\
\text { tive Per- } \\
\text { cent }\end{array}$ \\
\hline Undergraduate & 296 & 84.6 & 84.6 & 84.6 \\
Postgraduate & 33 & 9.4 & 9.4 & 94.0 \\
Diploma & 7 & 2.0 & 2.0 & 96.0 \\
Other & 14 & 4.0 & 4.0 & 100.0 \\
Total & 350 & 100.0 & 100.0 & \\
\hline
\end{tabular}

Table 6: Usage of devices for social media

\begin{tabular}{llll}
\hline & \multicolumn{2}{l}{ Responses } & Percent of Cases \\
& $\boldsymbol{N}$ & Percent & \\
\cline { 1 - 3 } Laptop & 138 & $31.1 \%$ & $39.4 \%$ \\
Desktop & 87 & $19.6 \%$ & $24.9 \%$ \\
Cell phone & 90 & $20.3 \%$ & $25.7 \%$ \\
Smartphone & 89 & $20.0 \%$ & $25.4 \%$ \\
IPad & 24 & $5.4 \%$ & $6.9 \%$ \\
Other & 16 & $3.6 \%$ & $4.6 \%$ \\
Total & 444 & $100.0 \%$ & $126.9 \%$ \\
\hline
\end{tabular}

\subsection{Descriptive statistics of construct}

Descriptive statistics of survey constructs are shown in table: 7 .

Table 7: Descriptive statistics of measured items of constructs

\begin{tabular}{ccccc}
\hline & N & Mean & Std. Deviation & Variance \\
\hline SM1 & 350 & 5.02 & 1.837 & 3.375 \\
SM2 & 350 & 4.97 & 1.764 & 3.111 \\
SM3 & 350 & 4.98 & 1.839 & 3.381 \\
SM4 & 350 & 4.79 & 1.910 & 3.647 \\
SM5 & 350 & 4.98 & 1.778 & 3.160 \\
PU1 & 350 & 4.75 & 1.883 & 3.545 \\
PU2 & 350 & 4.72 & 1.848 & 3.416 \\
PU3 & 350 & 4.68 & 1.882 & 3.541 \\
PU4 & 350 & 4.66 & 1.792 & 3.212 \\
PU5 & 350 & 4.70 & 1.868 & 3.489 \\
PU6 & 350 & 4.69 & 1.945 & 3.784 \\
PEOU1 & 350 & 4.88 & 1.839 & 3.380 \\
PEOU2 & 350 & 4.87 & 1.781 & 3.171 \\
PEOU3 & 350 & 4.81 & 1.900 & 3.609 \\
PEOU4 & 350 & 3.43 & 1.933 & 3.735 \\
PEOU5 & 350 & 4.78 & 1.831 & 3.351 \\
PEOU6 & 350 & 4.75 & 1.848 & 3.415 \\
CO1 & 350 & 4.71 & 1.844 & 3.399 \\
CO2 & 350 & 4.77 & 1.771 & 3.138 \\
CO3 & 350 & 4.73 & 1.851 & 3.425 \\
CO4 & 350 & 4.73 & 1.861 & 3.465 \\
CO5 & 350 & 4.57 & 1.895 & 3.593 \\
CO6 & 350 & 4.54 & 1.771 & 3.138 \\
SE1 & 350 & 4.87 & 1.799 & 3.236 \\
SE2 & 350 & 4.84 & 1.829 & 3.344 \\
SE3 & 350 & 4.74 & 1.794 & 3.220 \\
& & & & \\
\hline
\end{tabular}

\begin{tabular}{cllll}
\hline SE4 & 350 & 4.76 & 1.711 & 2.926 \\
RE1 & 350 & 5.10 & 1.761 & 3.101 \\
RE2 & 350 & 5.16 & 1.663 & 2.767 \\
RE3 & 350 & 5.12 & 1.743 & 3.038 \\
RE4 & 350 & 4.93 & 1.780 & 3.167 \\
CX1 & 350 & 4.82 & 1.878 & 3.525 \\
CX2 & 350 & 5.06 & 1.712 & 2.930 \\
CX3 & 350 & 4.89 & 1.895 & 3.593 \\
CX4 & 350 & 4.88 & 1.800 & 3.238 \\
Valid N & 350 & & &
\end{tabular}

SM: Intention to accept social media in education, PU: Perceived usefulness, PEOU: Perceived ease of use, CO: Compatibility, SE: Self efficacy, RE: Relevance, CX: Complexity

\subsection{Exploratory factor analysis}

Using SPSS, Principal components analysis (PCA) and exploratory factor analysis was performed.

\section{4. kmo and bartlett's test of sphericity}

KMO and BTS that demonstrates a .923 value of KMO measure of sampling adequacy and a $p<.001$ value for the former test, which showed suitability of data for executing analysis of factor in Table 8.

Table 8: Kmo and bartlett's test

\begin{tabular}{ccc}
\hline \multicolumn{3}{c}{ Kaiser-meyer-olkin measure of sampling } \\
adequacy. & $\mathbf{. 9 2 3}$ \\
\hline \multirow{2}{*}{ Bartlett's Test of } & Approx. Chi-Square & 11126.736 \\
Sphericity & Df & 595 \\
& Sig. & .000 \\
\hline
\end{tabular}

\subsection{Communalities}

Communalities items loaded on the EFA model which are given in Table 9.

Table 9: Communalities

\begin{tabular}{|c|c|c|}
\hline & Initial & Extraction \\
\hline SM1 & 1.000 & .836 \\
\hline SM2 & 1.000 & .802 \\
\hline SM3 & 1.000 & .824 \\
\hline SM4 & 1.000 & .828 \\
\hline SM5 & 1.000 & .843 \\
\hline PU1 & 1.000 & .790 \\
\hline PU2 & 1.000 & .795 \\
\hline PU3 & 1.000 & .811 \\
\hline PU4 & 1.000 & .805 \\
\hline PU5 & 1.000 & .824 \\
\hline PU6 & 1.000 & .836 \\
\hline PEOU1 & 1.000 & .797 \\
\hline PEOU2 & 1.000 & .815 \\
\hline PEOU3 & 1.000 & .780 \\
\hline PEOU4 & 1.000 & .638 \\
\hline PEOU5 & 1.000 & .798 \\
\hline PEOU6 & 1.000 & .818 \\
\hline $\mathrm{CO} 1$ & 1.000 & .786 \\
\hline $\mathrm{CO} 2$ & 1.000 & .774 \\
\hline $\mathrm{CO} 3$ & 1.000 & .779 \\
\hline $\mathrm{CO} 4$ & 1.000 & .783 \\
\hline $\mathrm{CO} 5$ & 1.000 & .805 \\
\hline $\mathrm{CO} 6$ & 1.000 & .719 \\
\hline SE1 & 1.000 & .715 \\
\hline SE2 & 1.000 & .787 \\
\hline SE3 & 1.000 & .776 \\
\hline
\end{tabular}




\begin{tabular}{lrr}
\hline SE4 & 1.000 & .775 \\
RE1 & 1.000 & .846 \\
RE2 & 1.000 & .667 \\
RE3 & 1.000 & .840 \\
RE4 & 1.000 & .843 \\
CX1 & 1.000 & .770 \\
CX2 & 1.000 & .649 \\
CX3 & 1.000 & .644 \\
CX4 & 1.000 & .636 \\
Extraction Method: Principal Component Analysis.
\end{tabular}

\subsection{Exploratory factor extraction model}

Kaiser's principle of Eigen values larger than 1 and the Screeplot was utilized to extract factors in table 10 .

Table 10: Total variance explained

\begin{tabular}{|c|c|c|c|c|c|c|c|c|c|}
\hline \multirow{2}{*}{ 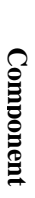 } & \multicolumn{3}{|c|}{ Initial eigenvalues } & \multicolumn{3}{|c|}{$\begin{array}{l}\text { Extraction Sums of } \\
\text { squared loadings }\end{array}$} & \multicolumn{3}{|c|}{$\begin{array}{l}\text { Rotation sums of } \\
\text { squared loadings }\end{array}$} \\
\hline & total & $\begin{array}{l}\% \text { of } \\
\text { vari- } \\
\text { ance }\end{array}$ & $\begin{array}{c}\text { cumu- } \\
\text { la- } \\
\text { tive } \%\end{array}$ & total & $\begin{array}{l}\% \text { of } \\
\text { vari- } \\
\text { ance }\end{array}$ & $\begin{array}{c}\text { cumu- } \\
\text { la- } \\
\text { tive } \%\end{array}$ & total & $\begin{array}{l}\% \text { of } \\
\text { vari- } \\
\text { ance }\end{array}$ & $\begin{array}{c}\text { cumula- } \\
\text { tive } \%\end{array}$ \\
\hline 1 & 13.7 & 39.3 & 39.3 & 13.7 & 39.3 & 39.3 & 4.9 & 14.2 & 14.2 \\
\hline 2 & 3.4 & 9.9 & 49.2 & 3.4 & 9.9 & 49.2 & 4.8 & 13.8 & 28.0 \\
\hline 3 & 2.7 & 7.7 & 57.0 & 2.7 & 7.7 & 57.0 & 4.7 & 13.6 & 41.6 \\
\hline 4 & 2.1 & 6.2 & 63.2 & 2.1 & 6.2 & 63.2 & 3.7 & 10.6 & 52.3 \\
\hline 5 & 1.9 & 5.4 & 68.7 & 1.9 & 5.4 & 68.7 & 3.1 & 9.0 & 61.3 \\
\hline 6 & 1.8 & 5.3 & 74.0 & 1.8 & 5.3 & 74.0 & 3.0 & 8.8 & 70.1 \\
\hline 7 & 1.3 & 3.7 & 77.8 & 1.3 & 3.7 & 77.8 & 2.6 & 7.6 & 77.8 \\
\hline \multicolumn{10}{|c|}{ Extraction Method: Principal Component Analysis. } \\
\hline
\end{tabular}

\subsection{Loadings of measured items on latent factors}

The rotated component matrix demonstrates loadings of individually calculated items on all of the 7 latent factors distinguished in EFA model in Table 11 and 12.

Table 11: Rotated component matrixa

\begin{tabular}{|c|c|c|c|c|c|c|c|}
\hline & \multicolumn{7}{|c|}{ Component } \\
\hline & 1 & 2 & 3 & 4 & 5 & 6 & 7 \\
\hline SM1 & & & & .748 & & & \\
\hline SM2 & & & & .742 & & & \\
\hline SM3 & & & & .743 & & & \\
\hline SM4 & & & & .776 & & & \\
\hline SM5 & & & & .799 & & & \\
\hline PU1 & .815 & & & & & & \\
\hline PU2 & .798 & & & & & & \\
\hline PU3 & .809 & & & & & & \\
\hline PU4 & .816 & & & & & & \\
\hline PU5 & .832 & & & & & & \\
\hline PU6 & .815 & & & & & & \\
\hline PEOU1 & & .833 & & & & & \\
\hline PEOU2 & & .849 & & & & & \\
\hline PEOU3 & & .859 & & & & & \\
\hline PEOU4 & & .774 & & & & & \\
\hline PEOU5 & & .842 & & & & & \\
\hline PEOU6 & & .842 & & & & & \\
\hline $\mathrm{CO} 1$ & & & .806 & & & & \\
\hline $\mathrm{CO} 2$ & & & .789 & & & & \\
\hline $\mathrm{CO} 3$ & & & .814 & & & & \\
\hline $\mathrm{CO} 4$ & & & .818 & & & & \\
\hline $\mathrm{CO} 5$ & & & .819 & & & & \\
\hline CO6 & & & .755 & & & & \\
\hline SE1 & & & & & & .742 & \\
\hline SE2 & & & & & & .785 & \\
\hline SE3 & & & & & & .826 & \\
\hline SE4 & & & & & & .836 & \\
\hline RE1 & & & & & .811 & & \\
\hline RE2 & & & & & .665 & & \\
\hline
\end{tabular}

\begin{tabular}{ccc}
\hline RE3 & .825 & \\
RE4 & .837 & .874 \\
CX1 & & .791 \\
CX2 & .793 \\
CX3 & .778 \\
CX4 & Extraction Method: Principal Component Analysis. \\
& Rotation Method: Varimax with Kaiser Normalization. \\
& a. Rotation converged in 6 iterations. \\
\hline \multicolumn{3}{c}{ Table 12: Reliability statistics } \\
\hline Cronbach's Alpha Cronbach's Alpha Based & N of Items \\
\hline \multicolumn{3}{c}{ on Standardized Items } \\
\hline
\end{tabular}

\subsection{Creation of latent factors}

The outcome of EFA suggested 7 latent factors were made by addition scores of all loaded items; now called the latent constructs in Table 13 and 14.

Table 13: Summary item statistics

\begin{tabular}{cccccccc}
\hline & Mean & $\begin{array}{c}\text { Mini- } \\
\text { mum }\end{array}$ & $\begin{array}{c}\text { Maxi- } \\
\text { mum }\end{array}$ & & & $\begin{array}{r}\text { RangeMax / Min Vari- } \\
\text { ance }\end{array}$ & $\begin{array}{c}\text { N of } \\
\text { Items }\end{array}$ \\
\hline Item Means & 4.79 & 3.42 & 5.16 & 1.73 & 1.50 & .079 & 35 \\
Item Variances & 3.33 & 2.76 & 3.78 & 1.01 & 1.36 & .056 & 35 \\
$\begin{array}{c}\text { Inter-Item } \\
\text { Covariances }\end{array}$ & 1.04 & -2.46 & 2.93 & 5.39 & -1.19 & .686 & 35 \\
$\begin{array}{c}\text { Inter-Item } \\
\text { Correlations }\end{array}$ & .317 & -.670 & .808 & 1.47 & -1.20 & .060 & 35 \\
\hline
\end{tabular}

Table 14: Item-total statistics

\begin{tabular}{|c|c|c|c|c|c|}
\hline & $\begin{array}{c}\text { Scale } \\
\text { Mean if } \\
\text { Item } \\
\text { Deleted }\end{array}$ & $\begin{array}{c}\text { Scale } \\
\text { Variance } \\
\text { if Item } \\
\text { Deleted }\end{array}$ & $\begin{array}{c}\text { Cor- } \\
\text { rected } \\
\text { Item- } \\
\text { Total } \\
\text { Corre- } \\
\text { lation }\end{array}$ & $\begin{array}{l}\text { Squa } \\
\text { red } \\
\text { Mul- } \\
\text { tiple } \\
\text { Cor- } \\
\text { rela- } \\
\text { tion }\end{array}$ & $\begin{array}{c}\text { Cronba } \\
\text { ch's } \\
\text { Alpha if } \\
\text { Item } \\
\text { Deleted }\end{array}$ \\
\hline SM1 & 162.65 & 1264.0 & .740 & .903 & .938 \\
\hline SM2 & 162.71 & 1271.1 & .714 & .813 & .938 \\
\hline SM3 & 162.70 & 1265.0 & .732 & .867 & .938 \\
\hline SM4 & 162.89 & 1264.8 & .704 & .887 & .938 \\
\hline SM5 & 162.70 & 1273.3 & .691 & .758 & .939 \\
\hline PU1 & 162.93 & 1270.7 & .669 & .746 & .939 \\
\hline PU2 & 162.95 & 1271.0 & .680 & .735 & .939 \\
\hline PU3 & 162.99 & 1266.9 & 699 & .760 & .938 \\
\hline PU4 & 163.02 & 1272.2 & .694 & .744 & .939 \\
\hline PU5 & 162.98 & 1270.2 & .679 & .763 & .939 \\
\hline PU6 & 162.99 & 1259.7 & .729 & .790 & .938 \\
\hline PEOU1 & 162.80 & 1286.7 & .561 & .760 & .940 \\
\hline PEOU2 & 162.81 & 1290.5 & .551 & .757 & .940 \\
\hline PEOU3 & 162.87 & 1297.4 & .461 & .701 & .941 \\
\hline PEOU4 & 164.25 & 1419.2 & -.404 & .530 & .948 \\
\hline PEOU5 & 162.89 & 1289.0 & .546 & .733 & .940 \\
\hline PEOU6 & 162.93 & 1284.5 & .575 & .772 & .940 \\
\hline CO1 & 162.97 & 1274.0 & .659 & .738 & .939 \\
\hline $\mathrm{CO} 2$ & 162.90 & 1277.5 & .659 & .716 & .939 \\
\hline $\mathrm{CO} 3$ & 162.95 & 1277.6 & .628 & .711 & .939 \\
\hline $\mathrm{CO} 4$ & 162.95 & 1276.6 & .632 & .703 & .939 \\
\hline CO5 & 163.11 & 1271.2 & .661 & .747 & .939 \\
\hline CO6 & 163.14 & 1279.3 & .645 & .676 & .939 \\
\hline SE1 & 162.81 & 1286.9 & .573 & .626 & .940 \\
\hline SE2 & 162.84 & 1284.3 & .583 & .690 & .939 \\
\hline SE3 & 162.94 & 1293.8 & .520 & .636 & .940 \\
\hline SE4 & 162.91 & 1301.7 & .482 & .615 & .940 \\
\hline RE1 & 162.57 & 1279.3 & .648 & .906 & .939 \\
\hline RE2 & 162.51 & 1291.8 & .582 & .684 & .940 \\
\hline RE3 & 162.56 & 1283.7 & .619 & .855 & .939 \\
\hline RE4 & 162.75 & 1284.5 & .599 & .879 & .939 \\
\hline CX1 & 162.86 & 1344.4 & .118 & .590 & .944 \\
\hline $\mathrm{CX} 2$ & 162.61 & 1340.0 & .169 & .444 & .943 \\
\hline CX3 & 162.79 & 1340.1 & .147 & .446 & .943 \\
\hline CX4 & 162.79 & 1329.1 & .242 & .477 & .942 \\
\hline RE1 & 162.57 & 1279.3 & .648 & .906 & .940 \\
\hline
\end{tabular}




\section{Conclusion}

Studies based on factors affecting user acceptance of social media in higher education institutions of Pakistan was motivated by remarkable advancement in technology. This appeared to be one of the foremost compulsions for change in the educational sector.

Various directions for future research, nevertheless, are unexplored. For instance, the results obtained are limited to students. Further research may utilize or replicate this work on faculty members and other institutions (schools and colleges). This may be constructive in ascertaining the external validity of the model. Additionally, it may be intriguing to apply this model to further cultural settings, such as other parts of Asia or the developed countries of the West. This may be helpful in offering evidence for the suitability of this model for different cultural settings. Understandably, the strength of this model may fluctuate across various cultural surrounding and thus demand experimentation. Social media usage facilitates the development of critical thinking among the student population and keeps them updated with the latest knowledge relevant to their educational interests. With the aid of social media teachers have the opportunity to create a productive educational setting for both students and other faculty members [20]. Further, students' learning skills can be enhanced through the usage of advanced teaching techniques that are media based.

\section{References}

[1] The Express Tribune Report. (2013). Retrieved October 1, 2015, from http://tribune.com.pk/story/567649/30m-internet-users-inpakistan-half-on-mobile-report/

[2] Fred D. Davis, Richard P. Bagozzii, Paul R. Warshaw. (1989). User Acceptance of Computer Technology: A Comparsion of Two Models. Management Science.

[3] Davis, F. D., Bagozzi, R. P., \& Warshaw, P. R. (1989). User acceptance of computer technology: a comparison of two theoretical models. Management Science, 35(8), 982-1003. Retrieved from http://www.jstor.org/stable/10.2307/2632151

[4] Rogers, E. M., Singhal, A., \& Quinlan, M. M. (1995). Diffusion of Innovations Everett M. Rogers. New York.

[5] Karahanna, E., W. Straub, D., \& L. Chervany, N. (1999). Information technology adoption across time: A cross-sectional comparsion of Pre-adoption and Post adoption beliefs. MIS Quarterly, 23(2), 183-213.

[6] Agarwal, R., Sambamurthy, V., \& Stair, R. M. (2000). The evolving relationship between general and specific computer efficacy. Information Systems Research.

[7] Davis, F. D. (1989). Perceived usefulness, Perceived ese of user and User acceptance of information technology. MIS Quarterly, 13(3), 319-340.

[8] Al-rahimi, W. M., Othman, M. S., \& Musa, M. A. (2013). Using TAM Model To Measure The Use Of Social Media For Collaborative Learning (Vol. 5, pp. 90-95). International Journal of Engineering Trends and Technology (IJETT).

[9] Agarwal, R., \& Prasad, J. (1999). Are Individual Differences Germane to the Acceptance of New Information Technologies? Decision Sciences, 30(2), 361-391. doi:10.1111/j.15405915.1999.tb01614.x

[10] KKwon, S. J., Park, E., \& Kim, K. J. (2014). What drives successful social networking services? A comparative analysis of user acceptance of Facebook and Twitter. The Social Science Journal, 111. doi:10.1016/j.soscij.2014.04.005

[11] Chang, S.-C., \& Tung, F.-C. (2007). An empirical investigation of students' behavioural intentions to use the online learning course websites. British Journal of Educational Technology, 39(1), 070625111823003-??? doi:10.1111/j.1467-8535.2007.00742.x

[12] HHardgrave, B., Davis, F. D., \& Riemenschneider, C. K. (2003). Investigating Determinants of Software Developers' Intentions to Follow Methodologies. Journal of Management Information Systems, 20(1), 123-151.

[13] PPituch, K. a., \& Lee, Y. (2006). The influence of system characteristics on e-learning use. Computers \& Education, 47(2), 222-244. doi:10.1016/j.compedu.2004.10.007

[14] SSu, L. T. (1994). The relevance of recall and precision in user evaluation. Journal of the American Society for Information Sci- ence, 45(3), 207-217 doi:10.1002/(SICI)1097 4571(199404)45:3<207::AID-ASI10>3.0.CO;2-1

[15] BBandura, A. (1986). Social Foundations of Thought and Action: A Social-Cognitive View. Prentice Hall:.Englewood Cliffs, NJ.

[16] KKoondhar, M. Y., Molok, N., Chandio, F., Rind, M. M., Raza, A., \& Shah, A. (2015). A Conceptual Framework for Measuring the Acceptance of Pervasive Learning. Proceedings of the 5th International Conference on Computing \& Informatics, (193), 97-103. Retrieved from <Go to ISI > :/WOS:000359431400013

[17] GGay, L. R., \& Airasian, P. W. (2000). Educational Research, Competencies for Analysis and Application (6th ed.). Merill an Imprint of Prentice Hall.

[18] SSekeran, U., \& Bougie, R. (2016). Research Methods for Business: A Skill-Building Approach (6th ed.). U.k: John Wiley \& Sons, Inc..

[19] MMoore, G., \& Benbasat, I. (1991). Development of an instrument to measure the perceptions of adopting an information technology innovation. Information Systems Research, 2, 192-222. doi:10.1287/isre.2.3.192

[20] RRaza, A., Chandio, F. H., Koondhar, M. Y., Rind, M. M., \& Shah, A. (2015). A Framework for the Analysis if Determinants of Socia Media Acceptance in Higher Educational Institutes of Pakistan. In the 5th International conference on computing and informatic, ICOCI 2015 (pp. 104-111). Istanbul, Turkey: Universiti Utara Malaysia. 